\title{
Caracterización mecánica y microestructural de aceros de baja activación candidatos como primera pared en los reactores de fusión por confinamiento magnético ${ }^{(\bullet)}$
}

\begin{abstract}
M.T. Hernández ${ }^{(*)}$, J. Lapeña ${ }^{(*)}$, G. de Diego ${ }^{(*)}$ y M. Schirra ${ }^{(* *)}$
Resumen Actualmente, se está estudiando de forma paralela al desarrollo del diseño de los reactores de fusión los posibles materiales a emplear en estos. Una de las cuestiones más críticas en esta investigación es la selección del material estructural a emplear como primera pared y envoltura. En el presente trabajo se estudian tres aleaciones de diseño alemán de bają activación, en las que se ha sustituido el niobio por tantalio o por cerio. Los resutados mecánicos muestran que las aleaciones, en las que se ha añadido cerio de forma controlada, están en la línea de las de baja activación existentes hasta ahora, pero la adición de tantalio presenta problemas al provocar una precipitación primaria y masiva de carburos $\mathrm{TaC}_{3}$ que desestabiliza la matriz y origina cambios microestructurales muy acusados. Así, ocurre un descenso en las propiedades mecánicas en torno a los $600{ }^{\circ} \mathrm{C}$, que la incapacita para la aplicación como primera pared, ya que la temperatura de trabajo de ésta se halla en torno a los $550{ }^{\circ} \mathrm{C}$.
\end{abstract}

Palabras clave: Aceros ferriticos. Fusión nuclear.

\section{Mechanical and microstructural characterization of low activation steels as first wall of nuclear fusion reactors}

\begin{abstract}
Currently, the the design development of fusion reactors and the possible materials to use in them are being studied in parallel. One of the most critical problems in this research is the structural material selection for the first wall and blanket. The aim of the present work is to study three low activation alloys designed in Germany in which niobium has been substituted by tantalum or cerium. The mechanical results show that the alloys containing cerium are in the same order of the low activation materials known to date, but the tantalum doped alloy produces $\mathrm{TaC}_{3}$ precipitation that destabilizes the matrix and provokes large microstructural changes. This causes a decrease of the mechanical properties at about $600{ }^{\circ} \mathrm{C}$. This fact makes this alloy insuitable for the first wall on fusion reactors, because the working temperature is near $550^{\circ} \mathrm{C}$.
\end{abstract}

Keywords: Ferritic steel. Nuclear fusion.

\section{INTRODUCCIÓN}

A nivel internacional se considera que el futuro reactor de fusión debe estar construido con materiales de baja activación. Este concepto incluye el

$(\bullet \quad$ Trabajo recibido el día 17 de julio de 1995 .

(*) Programa de Materiales, ITN, CIEMAT. Avda. Complutense, 22. 28040-Madrid (España).

(**) Forschungszentrum Karlsruche Institut für Materialforschung, FZK, 76021 Karlsruhe (R.F.A.). manejo fácil del material como residuo radiactivo, la seguridad en caso de incidencias y las operaciones de mantenimiento y reparación del reactor. En el primer caso, se intenta que los materiales que se hayan activado en el propio reactor puedan ser considerados como residuos de la clase $\mathrm{C}$ según la guía 10CFR61 de la NRC. Por otra parte, se trata de conseguir que los reactores de fusión no causen lesiones somáticas graves en caso de producirse un accidente. El último punto es el más difícil de lograr, ya que el nivel de radiación asociado a las 
reacciones nucleares es muy elevado y la tolerancia del cuerpo humano muy reducida. Una tasa de dosis de $5 \mathrm{mSv} / \mathrm{h}$ permite un contacto muy limitado en las operaciones de mantenimiento $(1 \mathrm{~h}$ al trimestre aproximadamente) y, aun así, para alcanzar este valor se requiere un decaimiento del material en un factor de $10^{6}$ a las pocas horas de la parada, con lo que se comprende que solamente un número muy limitado de materiales cumplen esta condición.

Los efectos de la irradiación sobre materiales están siendo muy estudiados (1), y aunque, en principio, se pensó que los aceros inoxidables podrían responder a las necesidades de la primera pared (2), se determinó que habría que ir al diseño de aleaciones especiales de baja activación reduciendo el contenido de elementos con isótopos de vida larga (3). Los aceros ferriticos-martensíticos son los más prometedores en este aspecto, debido principalmente a la alta resistencia al hinchamiento inducido por irradiación (4), aunque el mayor poblema que se puede encontrar es la caída de los parámetros mecánicos con el flujo neutrónico (5 y 6).

En el presente trabajo se presentan resultados de unas aleaciones denominadas genéricamente OPTIFER y que son consecuencia de una optimización del acero comercial DIN 1.4914, que se corresponde con una matriz martensítica de composición x18CrMoVNb12.

En una primera etapa se realizó un balance entre las propiedades más interesantes para la aplicación de la aleación comercial como primera pared: la tenacidad a la fractura y la fluencia a altas temperaturas. Así surgieron las primeras modificaciones del acero DIN 1.4914 que se denominó MANET (Martensitc Alloy for the Next European Torus) en el que se ajustó la relación $\mathrm{Al} / \mathrm{N}$ de forma que siempre el nitrógeno estuviera en exceso, dado el gran descenso que se producía en la resistencia a rotura y la disminución de los parámetros de fluencia si no era así. Además, con objeto de poder aplicar el diagrama de Schaeffler y eliminar la formación de ferrita $\delta$ de la microestructura, se realizó una estimación del equivalente $\mathrm{Cr}-\mathrm{Ni}$ por otros elementos menos comunes (Ta y Hf), llegándose a la conclusión de que porcentajes superiores al $5 \%$ en esta fase provocaban una caída en los parámetros mecánicos obtenidos en los ensayos de tracción. Estas modificaciones de composición sobre el material de partida originaron varias generaciones MANET (7 y 8). Además, dados los campos magnéticos tan elevados que se originarán en el reactor, podrían surgir problemas relacionados con su capacidad de magnetización.

Una vez lograda la optimización de la composición química de la aleación en cuanto a su comportamiento mecánico, fue necesario cumplir los criteros internacionales de baja activación (9). Mediante estudios de activación neutrónica y decaimiento es posible conocer qué elementos y en qué concentra- ciones pueden ser aceptables en una aleación para que tenga un tiempo de enfriamiento razonable. Así, se sustituyeron elementos que dan lugar a isótopos de vida larga como niobio, molibdeno y níquel por otros menos nocivos como tantalio y volframio (10).

En las aleaciones OPTIFER, además de cuidar todos los aspectos mencionados hasta aquí, se ha prestado una especial atención a aquellos elementos que, incluso a niveles de trazas, pueden ser muy perjudiciales desde el punto de vista radiológico, eliminando, por ejemplo, plata, terbio y holmio. Además, se han considerado las posibles reacciones secuenciales.

\section{MATERIALES}

Las aleaciones OPTIFER que se presentan en este trabajo están identificadas con la numeración 664, 666 y 667. La composición química está descrita en la tabla I en la que se puede observar que todás pertenecen al grupo de aceros denominados de baja aleación, en la que las concentraciones de elementos aleantes son mínimas. Según los cálculos neutrónicos realizados, las concentraciones máximas de los elementos que pueden ser aceptadas son las que se representan en la figura $1, \mathrm{y}$ así se han especificado en su diseño. Sin embargo, la sensibilidad de las técnicas de análisis disponibles hasta la fecha no permiten alcanzar estos niveles, detectando como máximo los valores representados

TABLA I.- Composición química de las aleaciones OPTIFER, $\%$ en peso

TABLE I.- Chemical composition of the OPTIFER alloys, \% weight

\begin{tabular}{|l|c|c|c|}
\hline & Aleación 664 & Aleación 666 & Aleación 667 \\
\hline $\mathrm{Cr}$ & 9,4 & 9,5 & 9,5 \\
$\mathrm{C}$ & 0,12 & 0,15 & 0,11 \\
$\mathrm{Mn}$ & 0,49 & 0,49 & 0,49 \\
$\mathrm{P}$ & 0,006 & 0,005 & 0,005 \\
$\mathrm{~S}$ & 0,006 & 0,003 & 0,002 \\
$\mathrm{~V}$ & 0,26 & 0,24 & 0,23 \\
$\mathrm{~B}$ & 0,0066 & 0,0082 & 0,0072 \\
$\mathrm{~W}$ & 0,96 & 0,02 & 0,96 \\
$\mathrm{Ce}$ & $<0,001$ & $<0,001$ & 0,034 \\
$\mathrm{Y}$ & - & - & $<0,001$ \\
$\mathrm{Ta}$ & 0,065 & 1,6 & 0,15 \\
$\mathrm{Si}$ & 0,07 & 0,07 & 0,08 \\
$\mathrm{Al}$ & 0,007 & 0,009 & 0,01 \\
$\mathrm{Cu}$ & 0,046 & 0,005 & 0,004 \\
$\mathrm{Ni}$ & 0,007 & 0,012 & 0,01 \\
$\mathrm{Mo}$ & 0,003 & 0,003 & 0,003 \\
$\mathrm{Nb}$ & 0,01 & 0,01 & 0,02 \\
$\mathrm{Ti}$ & 0,007 & 0,011 & 0,006 \\
$\mathrm{Sn}$ & $<0,001$ & $<0,001$ & $<0,001$ \\
$\mathrm{As}$ & $<0,001$ & $<0,001$ & $<0,01$ \\
\hline
\end{tabular}




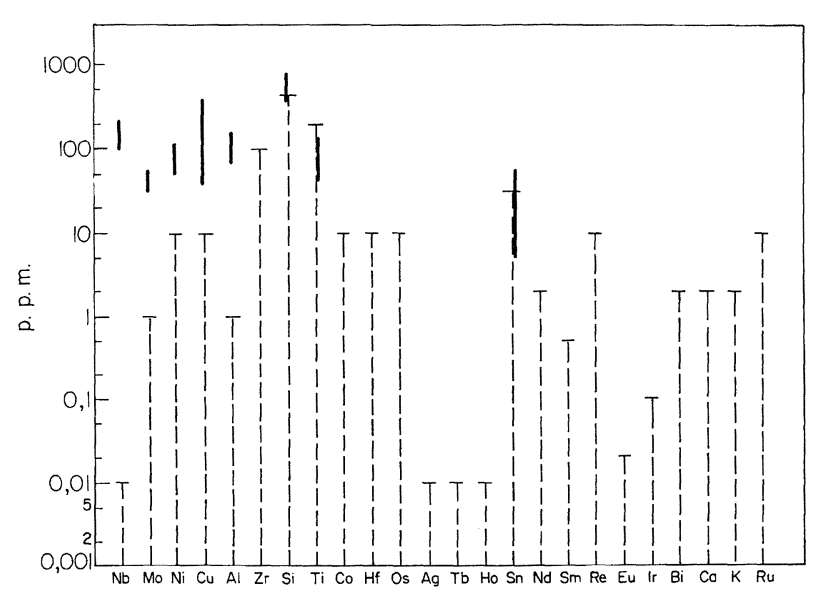

FIG. 1.- Concentraciones de elementos permitidos en las aleaciones de baja activación. En línea gruesa, se muestran los niveles medidos hasta el momento.

FIG. 1.- Element concentrations permited in the low activation alloys. The thicker line shows the level measured up to date.

en línea gruesa. Resulta imposible, por tanto, conocer si la concentración es inferior a la indicada. En el diseño se ha tenido especial cuidado en la relación atómica N/Al. La tabla II muestra el exceso de nitrógeno existente que previene la precipitación de ferrita $\delta$.

Las aleaciones 664 y 667 corresponden al mismo diseño base y se les ha añadido de forma controlada una pequeña cantidad de volframio. La única diferencia es el agente desoxidante utilizado en cada una de ellas: cerio en la primera e itrio en la segunda. Tradicionalmente, las desoxidaciones se suelen hacer con aluminio, pero en el caso de aleaciones de baja activación es imposible por tratarse de uno de los elementos prohibidos desde el punto de vista radiológico. La aleación 666 tiene una adición de tantalio para reducir el tamaño de grano y fue desoxidada con cerio.

No hubo ninguna dificultad en forjar las aleaciones y se prepararon barras de $25 \mathrm{~mm}$ de diámetro. El tratamiento posterior realizado fue una homogenización a $965^{\circ} \mathrm{C}$, seguido de un templado en aire a $1.075^{\circ} \mathrm{C}$ durante $30 \mathrm{~min}$, y un revenido posterior a $750{ }^{\circ} \mathrm{C}$ durante $2 \mathrm{~h}$. Dicho tratamiento se había utilizado previamente (9) para otras aleaciones de baja activación.

TABLA II.- Relación atómica N/Al en las aleaciones OPTIFER

TABLE II.- N/Al ratio in OPTIFER alloys

\begin{tabular}{|c|c|}
\hline Material & N/Al \\
\hline 664 & $+120 \mathrm{~N}$ \\
\hline 666 & $+121 \mathrm{~N}$ \\
\hline 667 & $+10 \mathrm{~N}$ \\
\hline
\end{tabular}

\section{MÉTODOS}

El análisis microestructural se ha llevado a cabo mediante microscopía óptica y electrónica de barrido, para lo que se pulieron las muestras hasta lograr una superficie especular. El microscopio de barrido utilizado es de la marca Hitachi mod. S-2500, trabaja a $20 \mathrm{kV}$ y lleva un sistema de microanálisis por energía dispersiva de rayos $\mathrm{X}$ acoplado al microscopio.

Las medidas de dureza se realizaron con un microdurómetro de la firma AKASHI, modelo HVK-FII, aplicando cargas de $300 \mathrm{~g}$ con un penetrador de punta de diamante sobre las superficies pulidas de las probetas, totalizándose 10 huellas por cada probeta. Estas medidas se contrastaron con medidas de dureza HV10 con un equipo Akashi mod. AVK-A siguiendo la norma ASTM E92. Las durezas HV30 se realizaron en la R.F.A.

Los ensayos de tracción se llevaron a cabo con una máquina universal de ensayos MTS mod. 810 con un horno vertical acoplado de forma que fuera posible trabajar en el rango de temperaturas 25-650 ${ }^{\circ} \mathrm{C}$. Las temperaturas se controlaron mediante tres termopares de Pt-Pt $10 \% \mathrm{Rh}$ acoplados al horno.

Los ensayos de fluencia se realizaron en máquinas que trabajan bajo carga constante, controlando la temperatura mediante tres termopares de Pt-Pt 10 $\% \mathrm{Rh}$, actuando cada uno sobre un tramo de calefacción, mediante un sistema informático específico que consigue variaciones máximas de $\pm 1{ }^{\circ} \mathrm{C}$. La extensometría se realiza por un sistema en paralelo en cada horno por medio de dos extensómetros, cuyas señales se tratan de forma que se puede obtener la curva de desplazamiento-tiempo si es necesario con resolución de $\pm 2 \mu \mathrm{m}$ en intervalos de un segundo. Se trabajó a $450-550{ }^{\circ} \mathrm{C}$ variando la carga entre 180 y $360 \mathrm{MPa}$.

Todos los equipos de ensayos mecánicos estaban calibrados por el laboratorio de metrología del CIEMAT, así como los termopares utilizados.

Las probetas empleadas en los ensayos de tracción y fluencia son roscadas, y sus dimensiones se dan en la figura 2.

\section{RESULTADOS Y DISCUSIÓN}

\subsection{Microestructura}

El estudio de las inclusiones y de los precipitados presentes tras el temple y revenido, se realizó mediante SEM-EDX. Los tres materiales se observaron previamente al ataque químico metalográfico para evitar la posible disolución de las inclusiones en los medios ácidos a los que se someten. De esta forma, únicamente dos de ellos mostraron inclusiones: el 666 y el 667. El aspecto de las inclusiones del material 666 viene representado en la figura 3a) 

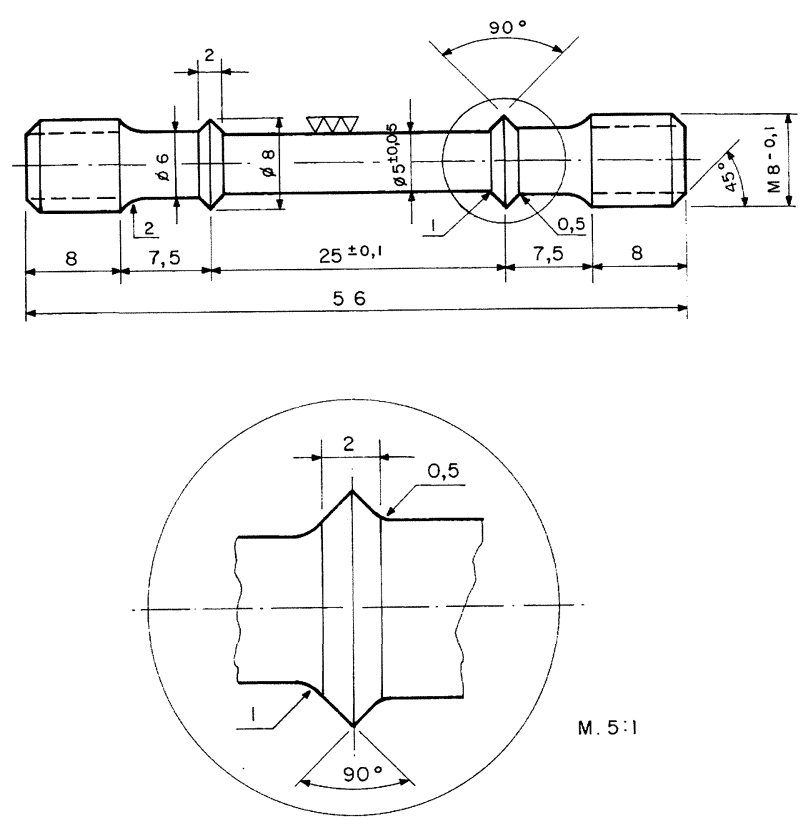

FIG. 2.- Probeta utilizada en los ensayos de tracción y de fluencia lenta.

FIG. 2.- Test piece used in tensile and creep tests.

en la que se aprecia que no presentan una forma única, pudiendo ser esféricas o alargadas. Son bastante pequeñas y no suelen superar nunca las $5 \mu \mathrm{m}$ de longitud, apareciendo aisladas o en pequeños grupos. Su análisis mediante EDX muestra claramente dos máximos a 1,804 , y $8,145 \mathrm{keV}$ típicos de la identificación de tantalio (Fig 3b) (Notaciones bibliográficas aseguran que se trata de $\mathrm{TaC}_{3}(6)$ ). Parecen ser bastante inertes desde el punto de vista químico, ya que perduran tras el ataque metalográfico. Este tipo de inclusiones es muy numeroso y constituye lo más característico del material. Además, tendrán una gran importancia en el comportamiento mecánico como se verá posteriormente.

Las inclusiones correspondientes al material 667 son bien diferentes a las descritas para la aleación anterior. Siempre presentan una forma similar: la de esfera agrietada y aislada, de 1,5 $\mu \mathrm{m}$ de diámetro aproximadamente (Fig. 4a). El análisis mediante EDX muestra, junto a los máximos de hierro y cromo, una señal a 4,84 keV (Fig. 4b), que puede asociarse al cerio. Estos agregados se disuelven fácilmente en medios ácidos, de forma que en las superficies pulidas y atacadas químicamente se presentan oquedades que corresponden a los agregados originales. Sin embargo, en este caso, la cantidad de inclusiones es muy pequeña y no se puede considerar representativa del material, ni asociarla a su comportamiento mecánico.

En las figuras 5a), 5b), y 5c), obtenidas por microscopía óptica, se observa la microestructura que presentan los materiales 664, 666 y 667 . El ataque de las superficies pulidas revela una clara estructura martensítica en los tres materiales. Es
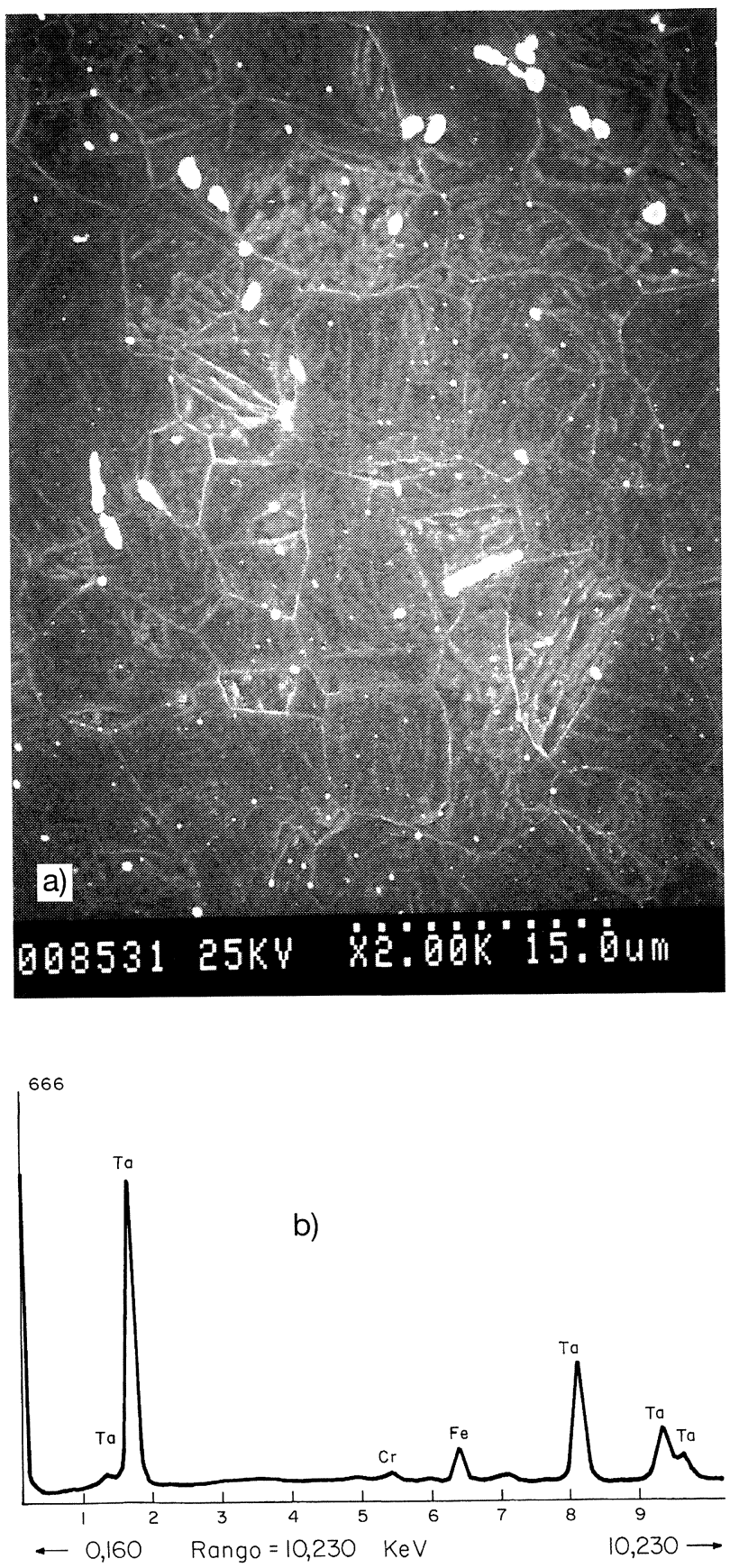

FIG. 3.- Material 666.

a) Inclusiones.

b) Análisis de las inclusiones mediante EDX.

FIG. 3.-Material 666.

a) Inclusions.

b) EDX inclusions analysis.

interesante destacar el efecto del tantalio como afinador de grano; como se puede apreciar en la figura 5b), el tamaño de grano del material 666 es muy inferior al de las restantes aleaciones.

Las obsevaciones realizadas con el microscopio electrónico de barrido permiten un conocimiento exhaustivo del efecto que ha tenido el tratamiento de temple + revenido aplicado a estos materiales. 

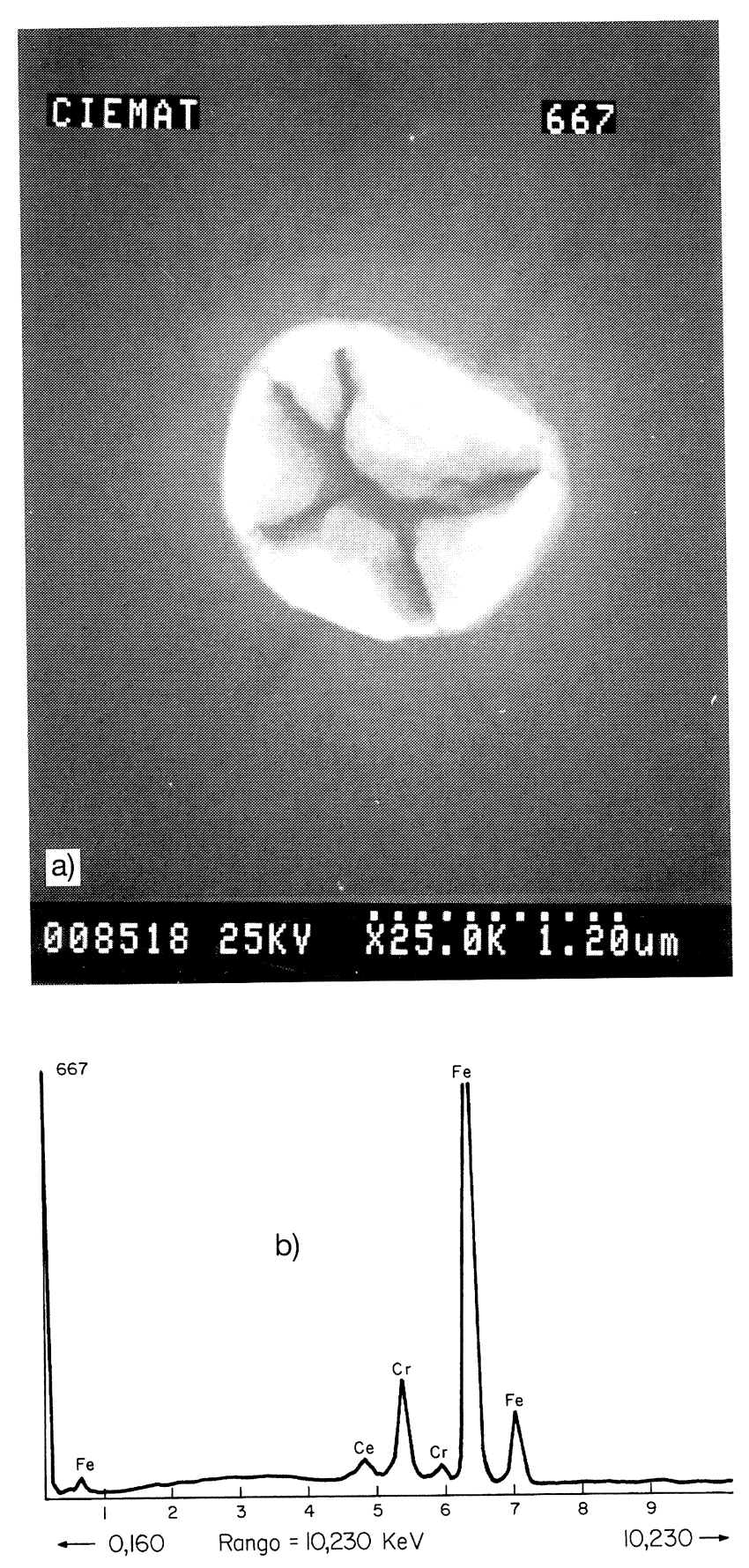

FIG. 4.- Material 667.

a) Inclusiones.

b) Análisis de las inclusiones mediante EDX.

FIG. 4.-Material 667.

a) Inclusions.

b) EDX inclusions analysis.

Las figuras 6a), 6b) y 6c) muestran los materiales 664666 y 667 que se caracterizan por presentar bordes de grano austeníticos con láminas de martensita y una gran cantidad de carburos de cromo típicos distribuidos especialmente a través de los bordes de grano, aunque existe también una gran cantidad en el interior. En ningún caso se ha podido detectar ferrita de forma evidente.
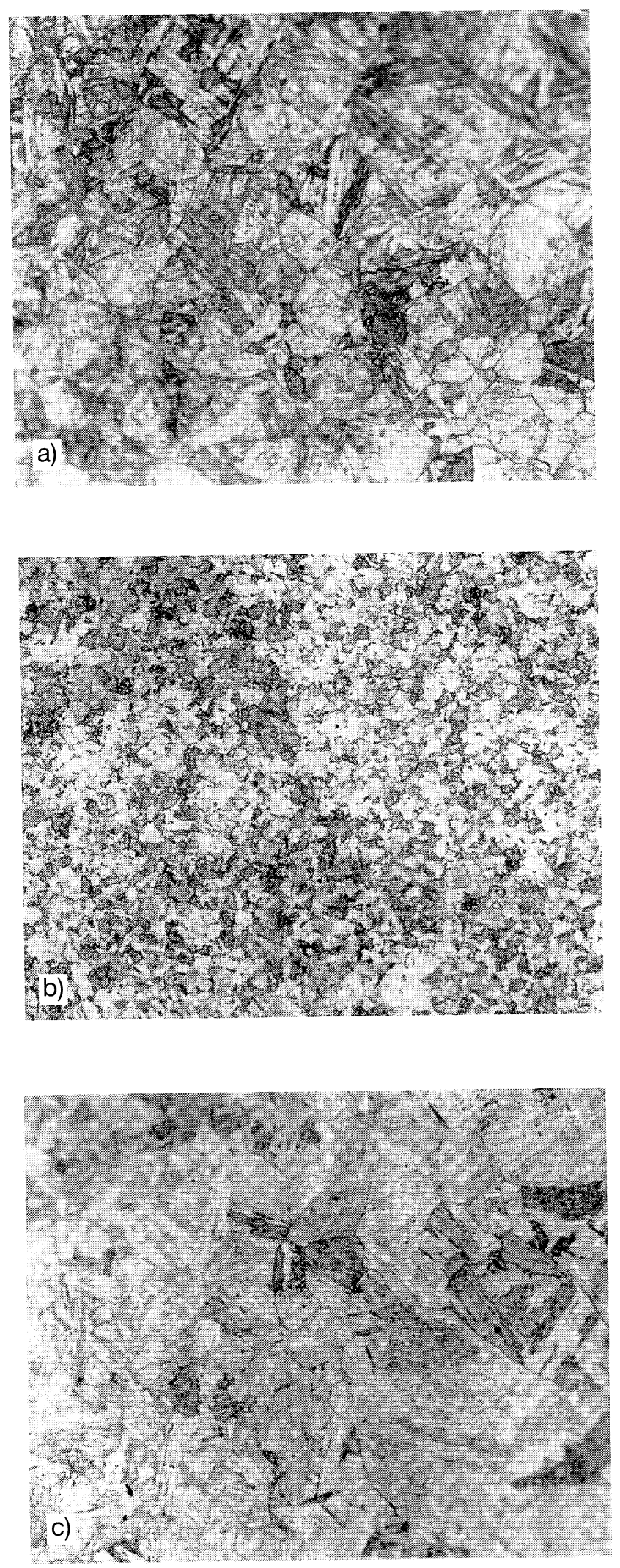

FIG. 5.- Microestructura tras el tratamiento a 1.075 ${ }^{\circ} \mathrm{C} / 30$ s $-750{ }^{\circ} \mathrm{C} / 2$ h. $\times 100$

a) Material 664. b) Material 666. c) Material 667.

FIG. 5.- Microstructure after treatment at 1.075 ${ }^{\circ} \mathrm{C} / 30$ s $-750{ }^{\circ} \mathrm{C} / 2 \mathrm{~h} . \times 100$

a) Material 664. b) Material 666. c) Material 667. 

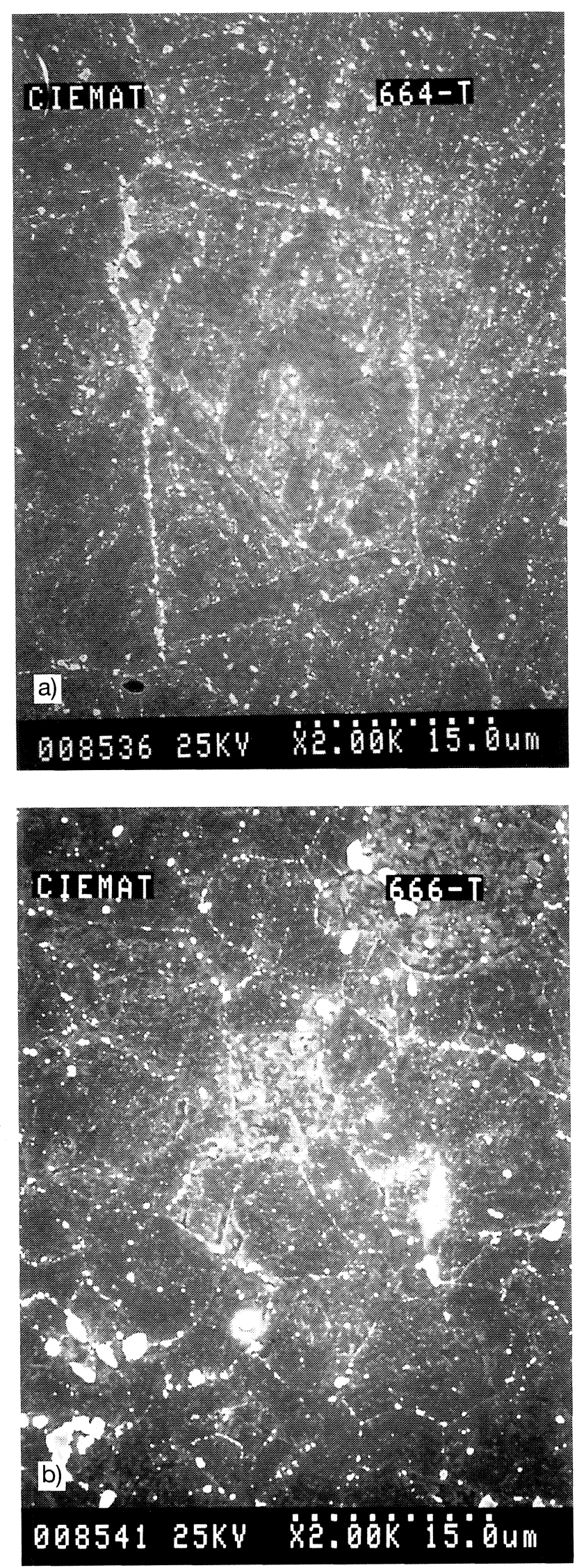

La variación del tamaño de grano en función del tratamiento de temple en doble vacío (v/v) aplicado se presenta en la figura 7.

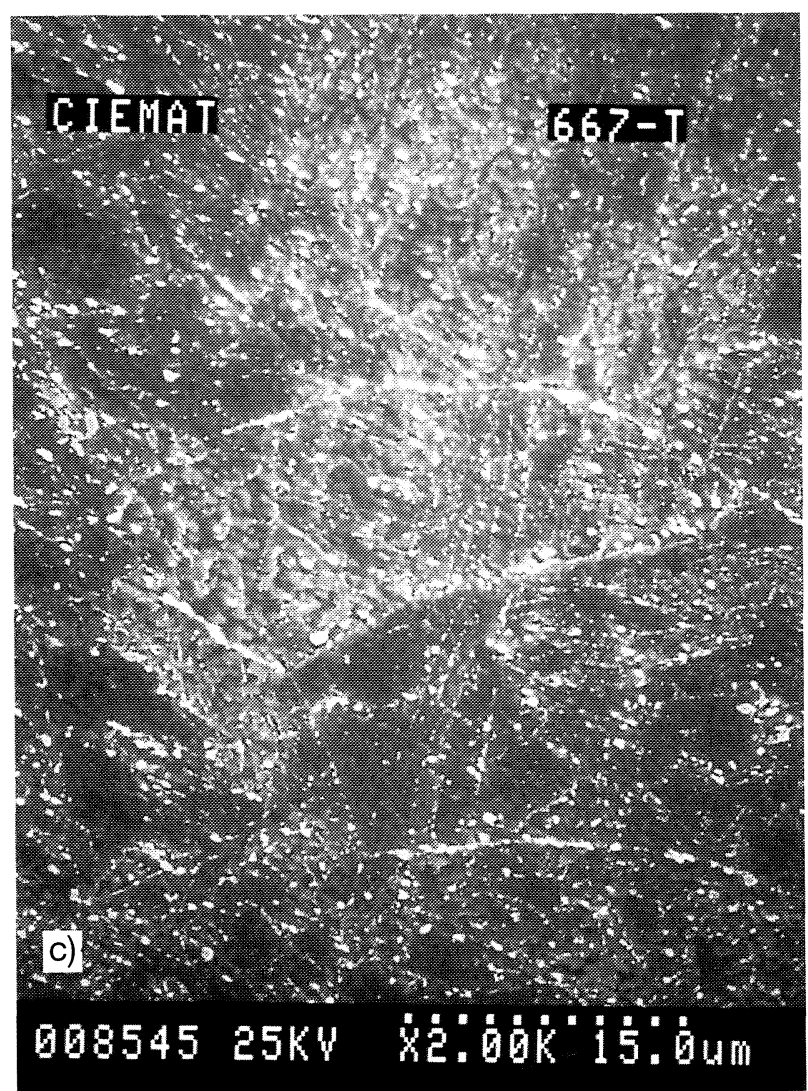

Fig. 6.- Microestructura tras el tratamiento a 1.075 ${ }^{\circ} \mathrm{C} / 30 \mathrm{~s}-750{ }^{\circ} \mathrm{C} / 2 \mathrm{~h}$ (SEM).

a) Material 664. b) Material 666. c) Material 667.

FIG. 6.- Microstructure after treatment at 1.075 ${ }^{\circ} \mathrm{C} / 30$ s $-750{ }^{\circ} \mathrm{C} / 2 \mathrm{~h}$ (SEM).

\subsection{Dureza}

La variación de la dureza en función de los tratamientos de temple $\left(950-1.150{ }^{\circ} \mathrm{C}\right)$ y revenido $(300-$ $900{ }^{\circ} \mathrm{C}$ ) se muestra en las figuras 8 y 9 . Estos tratamientos se realizaron en doble vacío para evitar

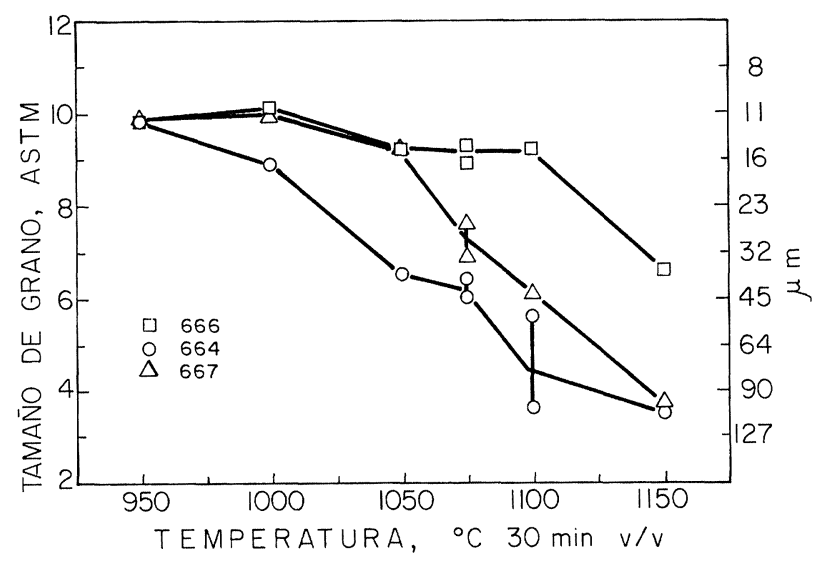

FIG. 7.- Variación del tamaño de grano con la temperatura de temple para los tres materiales.

FIG. 7.- Grain size vs. tempering temperature for the three alloys. 


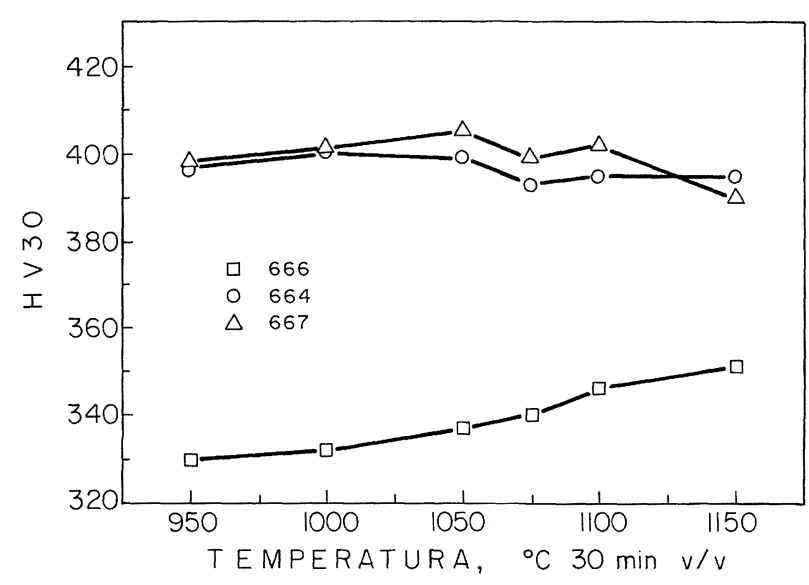

FIG. 8.- Variación de la dureza con la temperatura de temple para los tres materiales.

FIG. 8.- Hardness vs. tempering temperature for the three alloys.

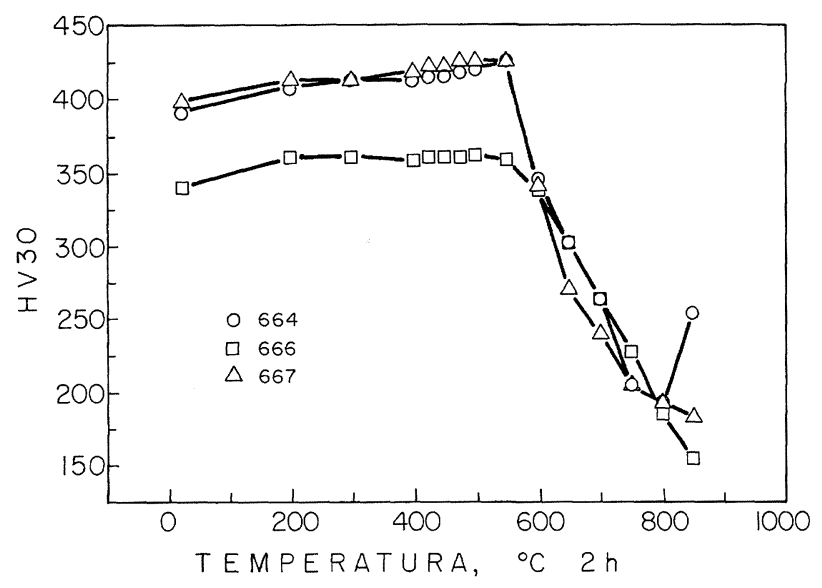

Fig. 9.- Variación de la dureza Vickers con la temperatura de revenido para los tres materiales.

FIG. 9.- Vickers hardness vs. annealing temperature for the three alloys.

oxidaciones. Aunque las tres aleaciones presentan valores altos de dureza, se puede apreciar que la 666 es sensiblemente inferior a las otras dos. La explicación es sencilla si se tiene en cuenta la precipitación primaria de $\mathrm{TaC}_{3}$ que sufre esta aleación y que retira gran parte del carbono de la solución sólida. Igualmente, no es posible encontrar la subida de dureza típica en aceros martensíticos que ocurre en los tratamientos de revenido en torno a $700{ }^{\circ} \mathrm{C}$, dado que el contenido de carbono real de la matriz es menor al diseñado originalmente.

\subsection{Ensayos de tracción}

Los resultados de los ensayos de tracción se muestran en las figuras 10a) y 10b), en las que se representa la resistencia mecánica y el límite elásti- co como función de la temperatura del ensayo. Puede apreciarse la disminución de ambos parámetros al aumentar la temperatura como corresponde a materiales dúctiles. Los valores de la aleación 666 siempre aparecen superiores a los de las otras dos para las mismas condiciones de ensayo, mientras que los de la 664 se presentan inferiores. Las figuras 10c) y 10d) representan los valores del alargamiento y reducción de área sufrido en las aleaciones tras estos ensayos.

Una representación muy útil en este tipo de experimentos es la que se muestra en la figura 11 . Se trata de representar la relación entre el límite elástico y la resistencia mecánica en función de la temperatura de trabajo, de forma que es posible obtener información sobre la resistencia que puede tener el material sin que sufra deformaciones plásticas. Como se ve en la figura, se mantiene la secuencia de los valores en los materiales, es decir, la aleación 666 presenta los valores más altos, siendo los menores los encontrados en el 667, apareciendo, además, un máximo muy acusado en torno a 500 ${ }^{\circ} \mathrm{C}$. El efecto'se debe a las características propias de cada aleación, pero es un hecho importante que este máximo se presente, precisamente, en torno a 500 ${ }^{\circ} \mathrm{C}$, que será la temperatura de trabajo de la primera pared.

Tras los ensayos, se observaron las superficies de fractura de las probetas pudiéndose comprobar que se trataba de fracturas totalmente dúctiles ocurridas como consecuencia de la coalescencia de granos en todo el rango de temperaturas de trabajo. El aspecto de las superficies de rotura viene representado en las figuras $12 \mathrm{a}), 12 \mathrm{~b}$ ) y 12c) para los materiales 664,666 , y 667 , respectivamente, ensayados a $300{ }^{\circ} \mathrm{C}$.

\subsection{Envejecimiento térmico}

Se han realizado tratamientos de envejecimiento térmico desde el estado de recepción, en el intervalo de temperaturas de $500-700{ }^{\circ} \mathrm{C}$, durante 20,200 y $2.000 \mathrm{~h}$. Los resultados se representan en función del parámetro de Haffe-Hollomon que incluye tiempo y temperatura de envejecimiento. En la figura 13 se puede observar que, mientras para las aleaciones 664 y 667 las curvas decaen suavemente para valores altos del parámetro, el material 666 sufre una caída brusca hacia $\mathrm{P} \sim 19$ aproximadamente. En efecto, dadas las características especiales de la aleación 666 que se han comentado anteriormente, resulta ser más inestable que las otras dos y sufre un claro proceso de recristalización cuando se la somete durante un tiempo a temperaturas superiores a $600{ }^{\circ} \mathrm{C}$. Este proceso se refleja en las figuras 14a), 14b) y 14c) que representan el material con 

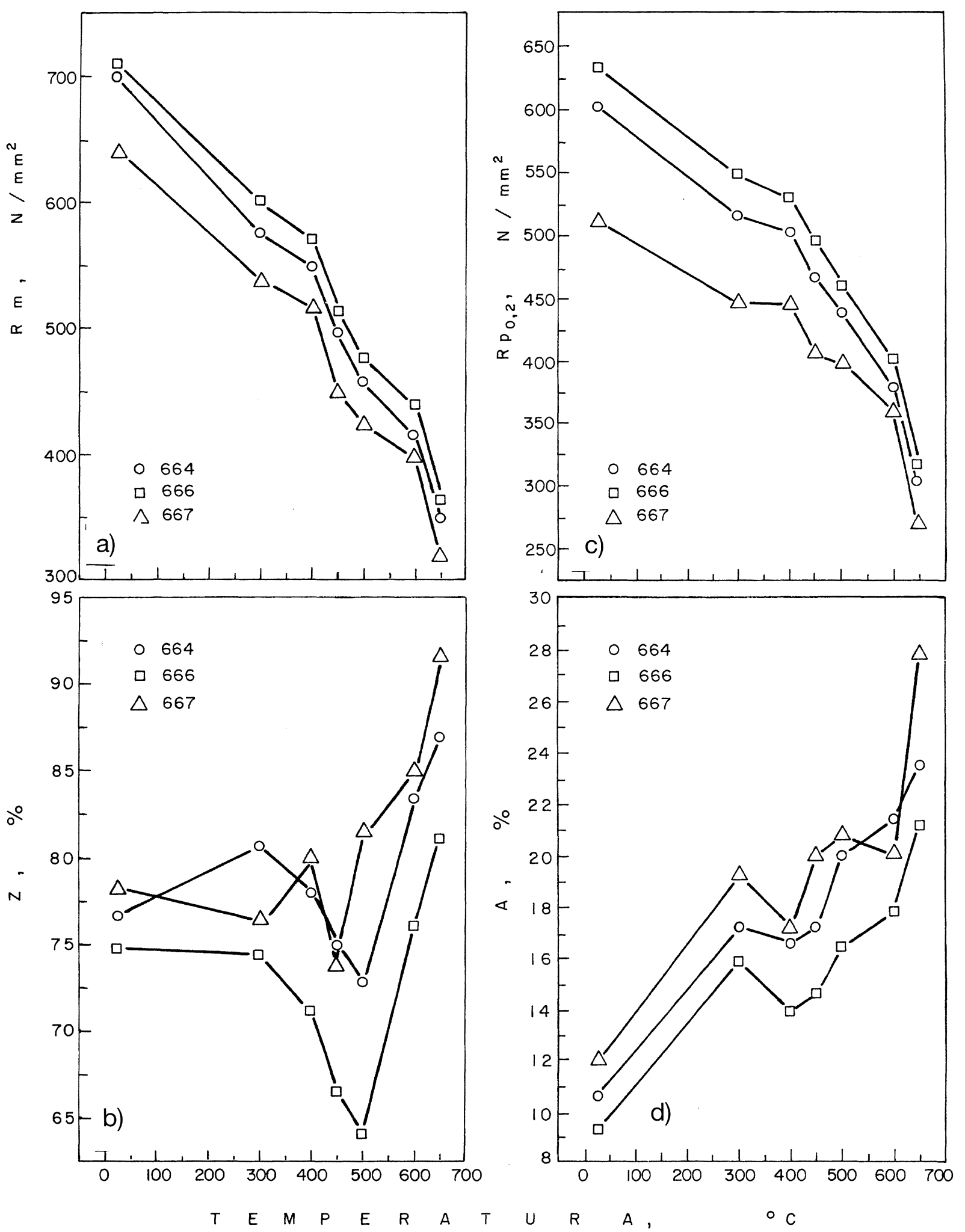

FIG. 10.- Aleaciones OPTIFER.

a) Resistencia mecánica en función de la temperatura de ensayo.

b) Reducción de área en función de la temperatura de ensayo.

c) Límite elástico en función de la temperatura de ensayo.

d) Alargamiento en función de la temperatura de ensayo.

FIG. 10.-OPTIFER alloys.

a) Tensile strength vs. test temperature. b) Reduction of area vs. test temperature.

c) Yield strength vs. test temperature. d) Elongation decrease vs. test temperature. 


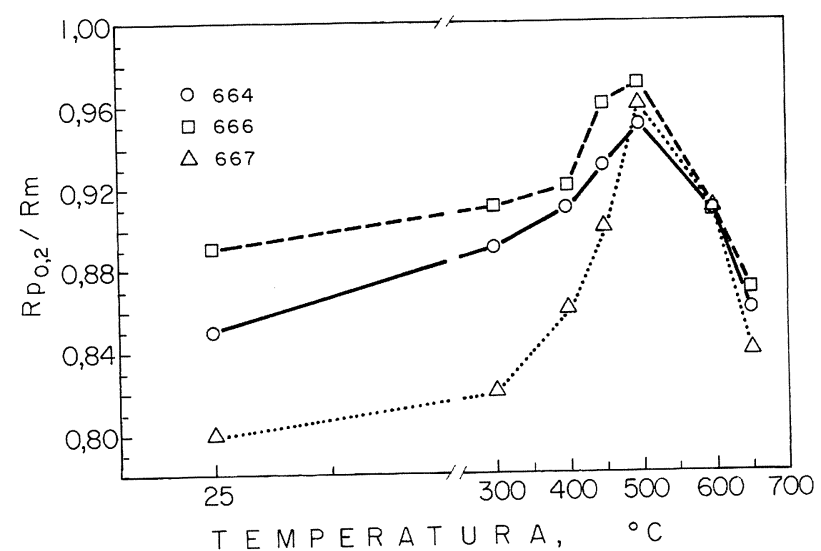

FIG. 11.- Relación límite elástico/resistencia mecánica para las aleaciones OPTIFER.

FIG. 11.- Yield strength/ultimate stress ratio for the OPTIFER alloys.

tratamientos de envejecimiento de $500{ }^{\circ} \mathrm{C} / 2.000 \mathrm{~h}$, $600{ }^{\circ} \mathrm{C} / 2.000$ h y $700{ }^{\circ} \mathrm{C} / 2.000 \mathrm{~h}$ en el que se aprecia un crecimiento de grano desmesurado para el último. Como consecuencia de ese cambio microestructural tan acusado, ocurre una variación muy importante en la dureza, que disminuye, tras la rrecristalización, desde valores de HV30 de 234 hasta

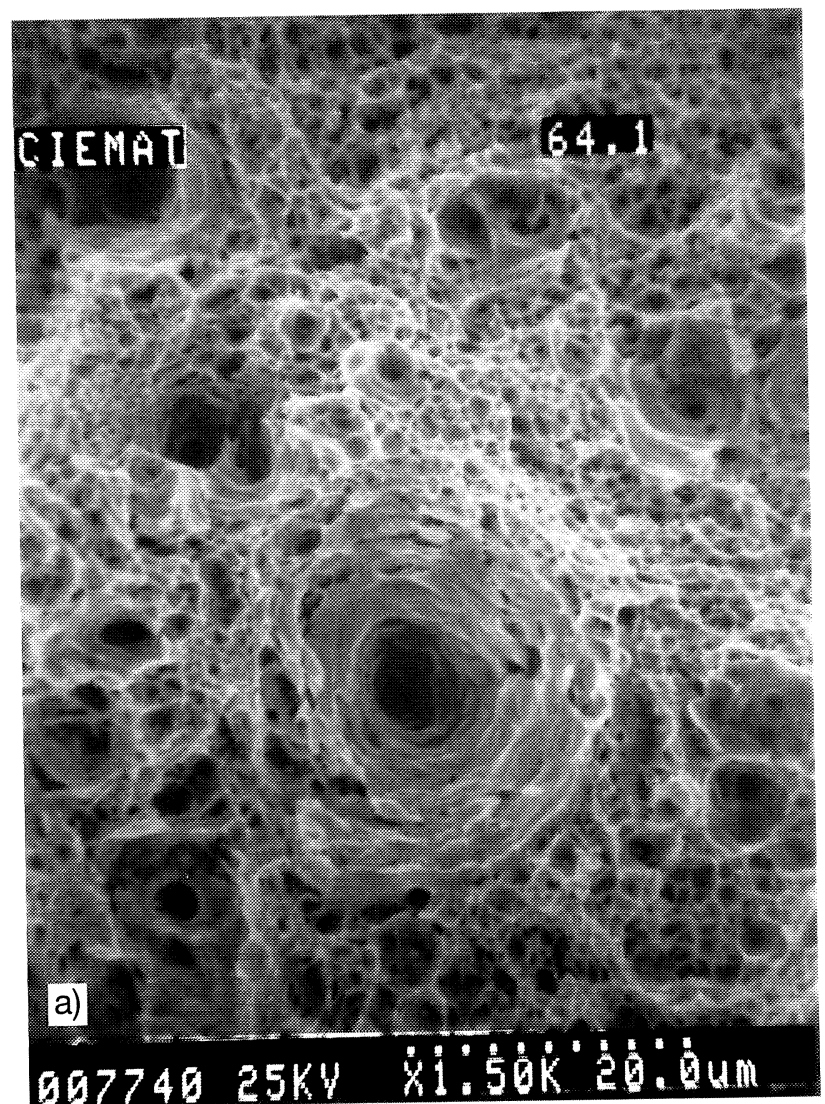

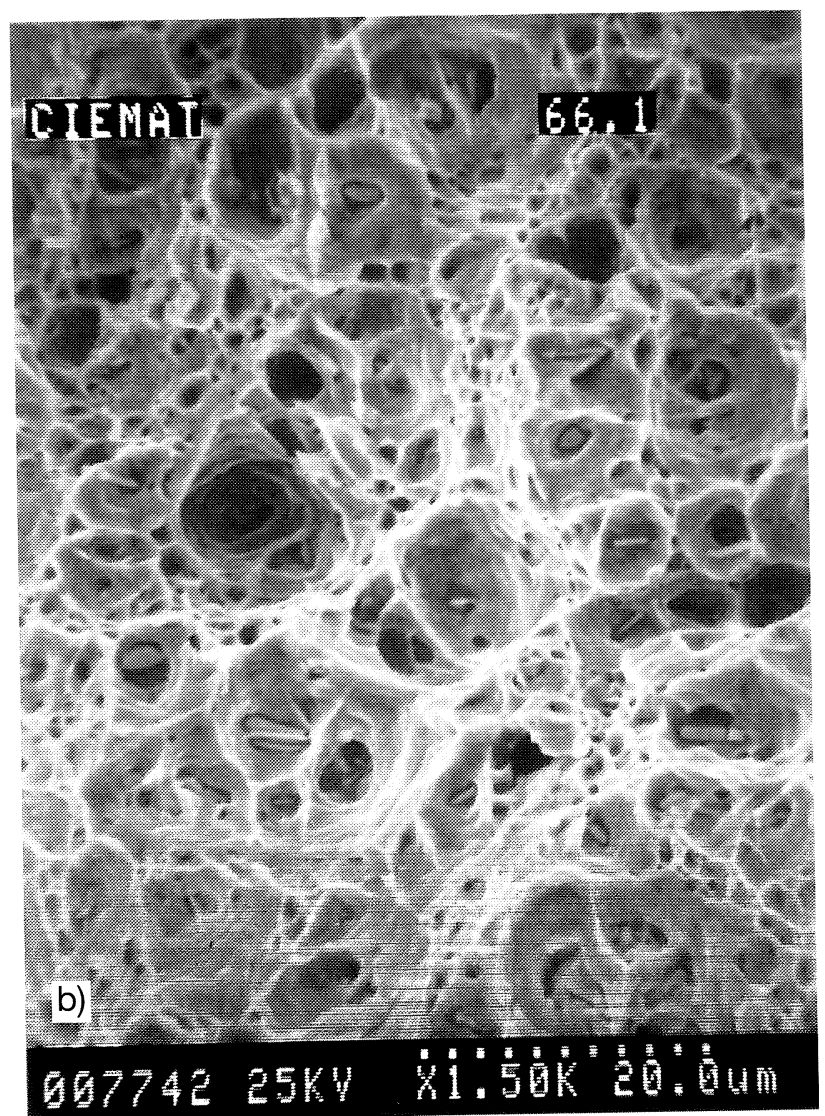

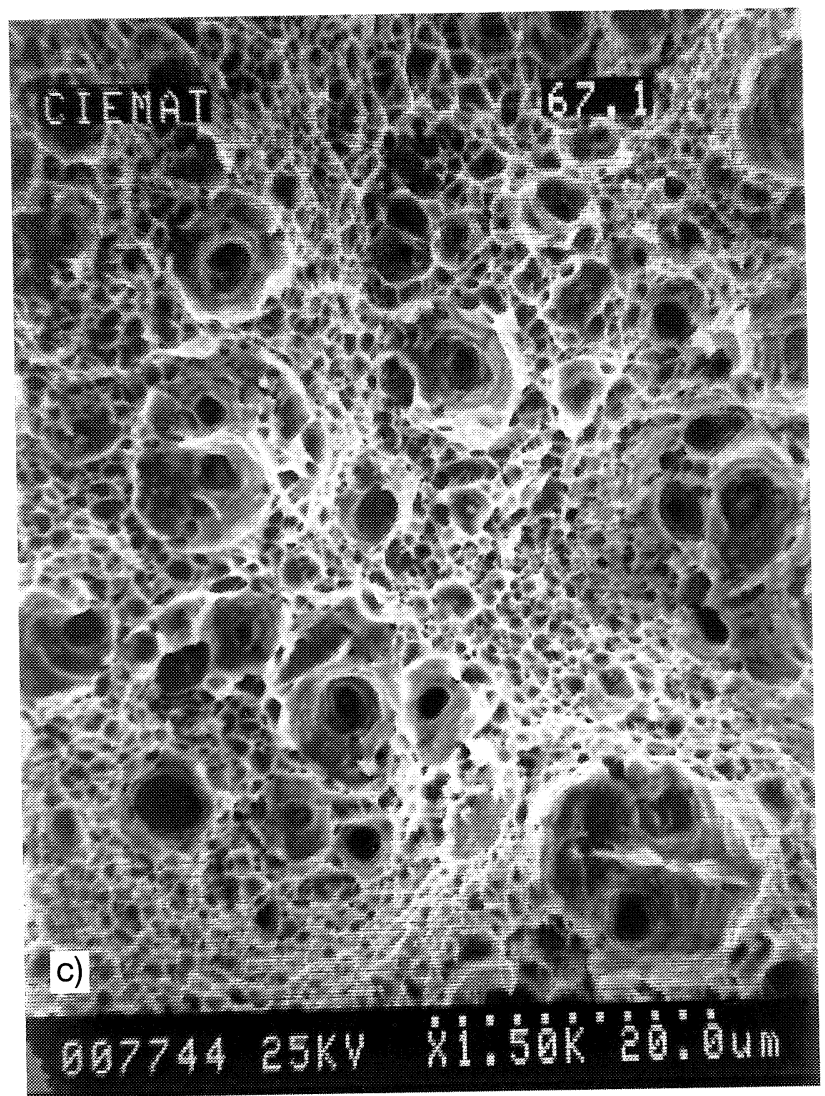

FIG. 12.- Imagen de las superficies de fractura tras los ensayos de tracción. a) Aleación 664. b) Aleación 666. c) Aleación 667.

FIG. 12.-Fresh fracture after tensile tests.

a) Aleación 664. b) Aleación 666. c) Aleación 667. 


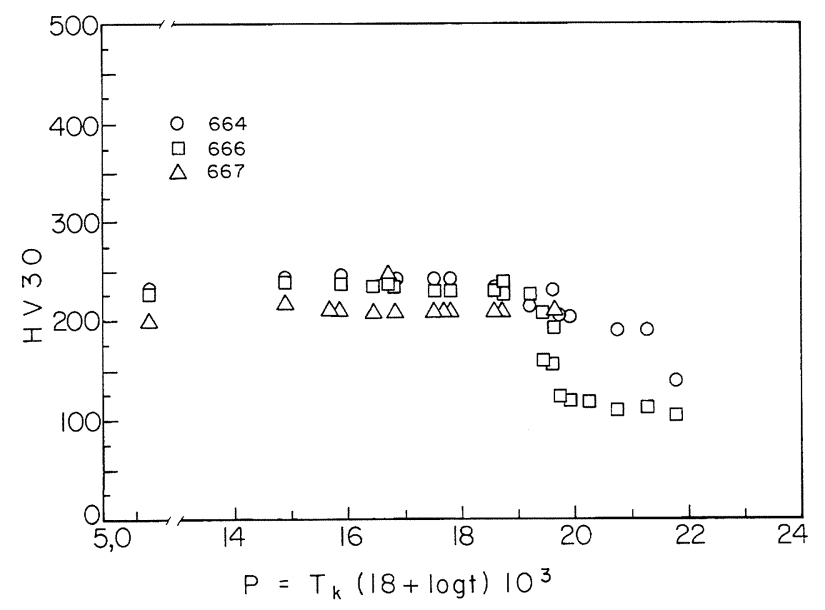

FIG. 13.- Variación de la dureza Vickers en función del parámetro de Haffe-Hollomon.

FIG. 13.- Vickers hardness vs. Haffe-Hollomon parameter.

110. Sin embargo, en el resto de las aleaciones, la microestructura es mucho más estable y permanece prácticamente inalterada tras los tratamientos suministrados. Únicamente, para la temperatura más alta utilizada $\left(700{ }^{\circ} \mathrm{C}\right)$ y el tiempo mayor $(2.000 \mathrm{~h})$, se encuentra una pequeña diferencia con el estado original en cuanto a la distribución de carburos (Figs. $15 a), 15 b)$ y $15 c)$. La gran estabilidad microestructural encontrada en las aleaciones 664 y 667 con los tratamientos térmicos es la responsable de que la dureza permanezca tan constante en todo el rango de temperaturas y tiempo explorados.

\subsection{Ensayos de fluencia}

Los resultados de fluencia se suelen representar en función del parámetro de Larson-Miller (11), que engloba la temperatura del ensayo y el tiempo hasta rotura. De esta forma, es posible disponer de información sobre muchas situaciones diferentes, de forma conjunta, y hacer predicciones sobre el tiempo de vida en servicio de un material determinado. Las cargas aplicadas a cada probeta están en función de las temperaturas a las que se han sometido, siendo menores a temperaturas más altas.

Las curvas de fluencia que se están obteniendo no presentan ninguna anomalia destacable, aumentando la velocidad secundaria de fluencia a medida que la carga aplicada es mayor para la misma temperatura de ensayo. Únicamente el material 666 presenta de forma ocasional algún "salto" que posiblemente esté relacionado con los procesos de recristalización que se observan en su microestructura sobre $550^{\circ} \mathrm{C}$.

Según se puede observar en la figura 16 , estas curvas decaen para valores altos del parámetro en
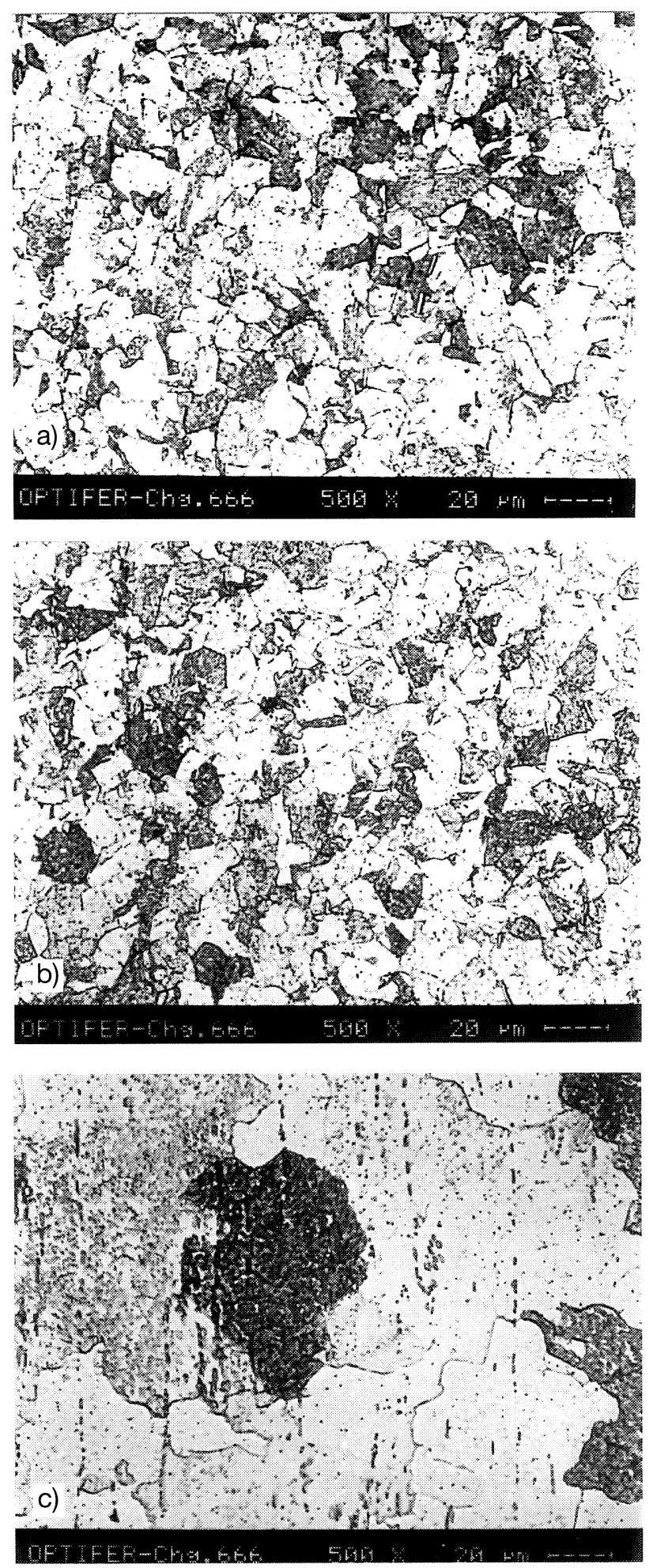

FIG. 14.- Microestructura del material 666 tras un tratamiento de envejecimiento. $\times 500$.

a) $500{ }^{\circ} \mathrm{C} / 2.000 \mathrm{~h} \mathrm{HV}: 234$.

b) $600{ }^{\circ} \mathrm{C} / 2.000 \mathrm{~h} \mathrm{HV}: 230-233$.

c) $700{ }^{\circ} \mathrm{C} / 2.000 \mathrm{~h} \mathrm{Hv}: 107-110$.

FIG. 14.- Microstructure of the material 666 after the ageing treatment. $\times 500$.

a) $500{ }^{\circ} \mathrm{C} / 2.000 \mathrm{~h} \mathrm{HV}: 234$.

b) $600{ }^{\circ} \mathrm{C} / 2.000 \mathrm{~h} \mathrm{HV}: 230-233$.

c) $700{ }^{\circ} \mathrm{C} / 2.000 \mathrm{~h} \mathrm{HV}: 107-110$. 

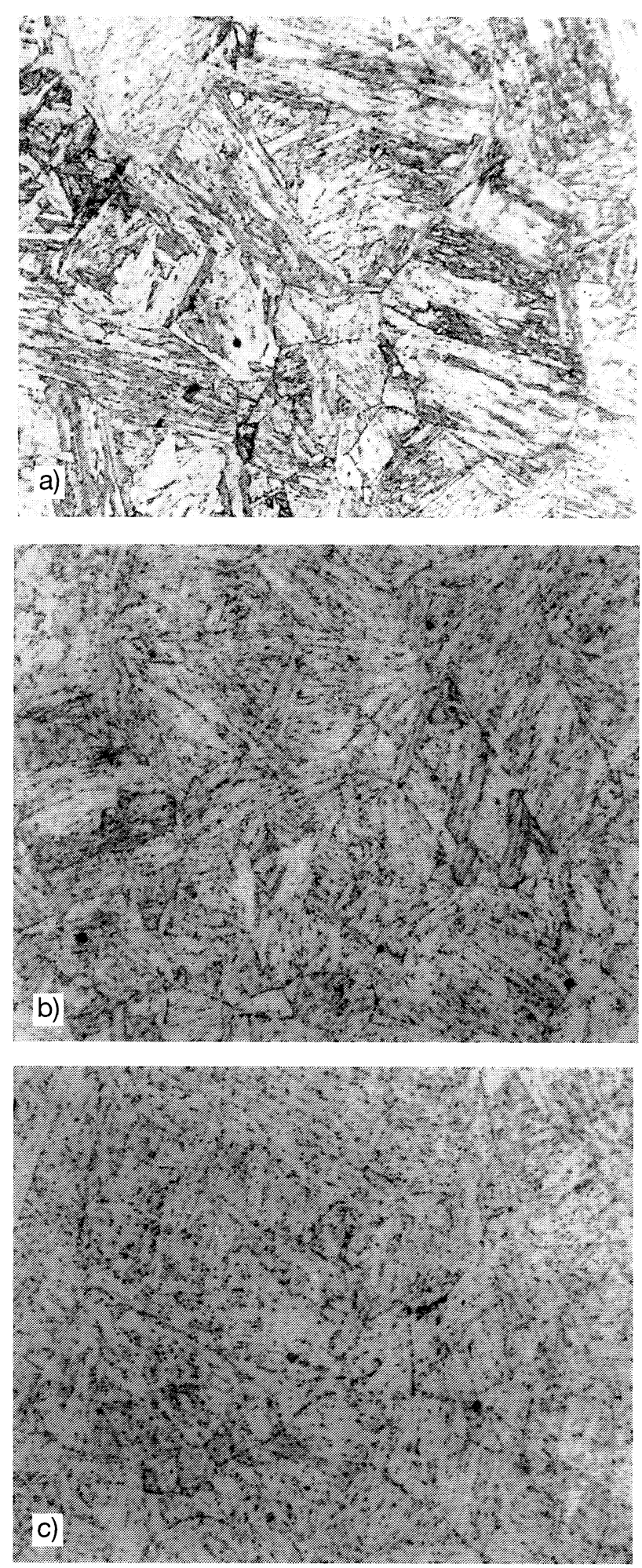

FIG. 15.- Microestructura de la aleación 667 tras un tratamiento de envejecimiento. $\times 500$.
a) $700{ }^{\circ} \mathrm{C} / 20 \mathrm{~h}$.
b) $700{ }^{\circ} \mathrm{C} / 200 \mathrm{~h}$.
c) $700{ }^{\circ} \mathrm{C} / 2.000 \mathrm{~h}$.

FIG. 15.-Microstructure of the material 667 after the ageing treatment. $\times 500$.
a) $700{ }^{\circ} \mathrm{C} / 20 \mathrm{~h}$.
b) $700{ }^{\circ} \mathrm{C} / 200 \mathrm{~h}$.
c) $700{ }^{\circ} \mathrm{C} / 2.000 \mathrm{~h}$.

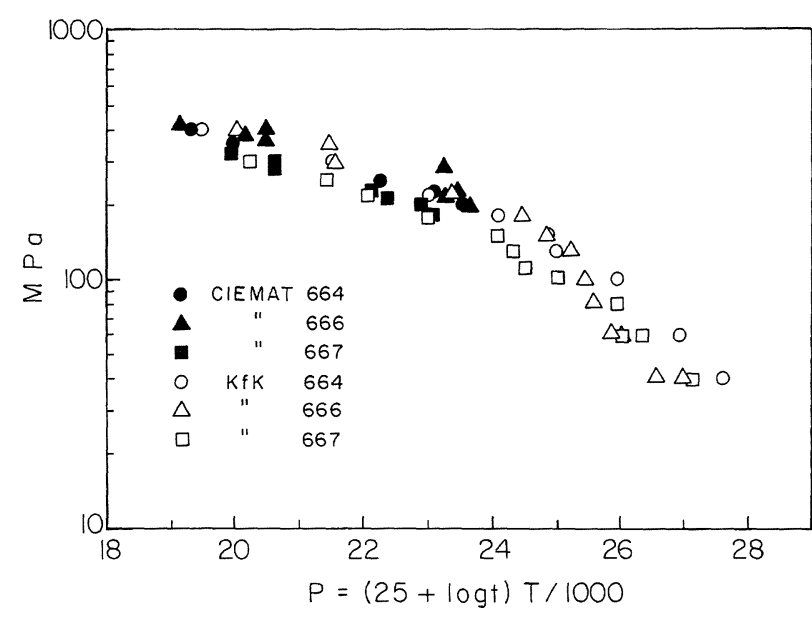

FIG. 16.- Comportamiento a fluencia de las aleaciones OPTIFER.

FIG. 16.-Creep behaviour of the OPTIFER alloys.

los materiales 664 y 667, lo que supone un comportamiento normal. La curva correspondiente al material 666 presenta una disminución muy abrupta para valores del parámetro comprendidos entre 24 y 25. Este fenómeno se puede justificar, al igual que la caída en la dureza, debido a la recristalización (aumento de tamaño de grano) que sufre el material a temperaturas superiores a $600{ }^{\circ} \mathrm{C}$, lo que limita su utilización de forma importante (Figs. 17a), 17b), 17c) y $17 d$ ).

Es interesante destacar el efeto que presentan las inclusiones de tantalio en el proceso de rotura del material 666. Como se puede ver en la figura 18 , cerca de la zona de rotura se pueden observar multitud de grietas que se generan en la interfase inclusión-matriz. Posiblemente, esta sea la causa de las pequeñas anomalías que se encuentran ocasionalmente en las curvas de fluencia de esta aleación, en comparación con las otras dos en las que estas microgrietas no se han detectado (Fig. 19).

Aunque el material 666 presenta una resistencia mecánica y límite elástico mayores que los otros dos, su utilización está bastante limitada como consecuencia de la inestabilidad de su microestructura y de los procesos de recristalización que sufre. Hay que tener en cuenta que, para el tipo de aplicación que se pretende, resultan mucho más interesantes los ensayos de fluencia lenta que los de tracción. Las aleaciones 664 y 667 presentan un comportamiento muy similar, aunque la 664 consigue valores ligeramente superiores.

\section{CONCLUSIONES}

- Las aleaciones OPTIFER suponen un paso importante en el desarrollo de materiales de baja 

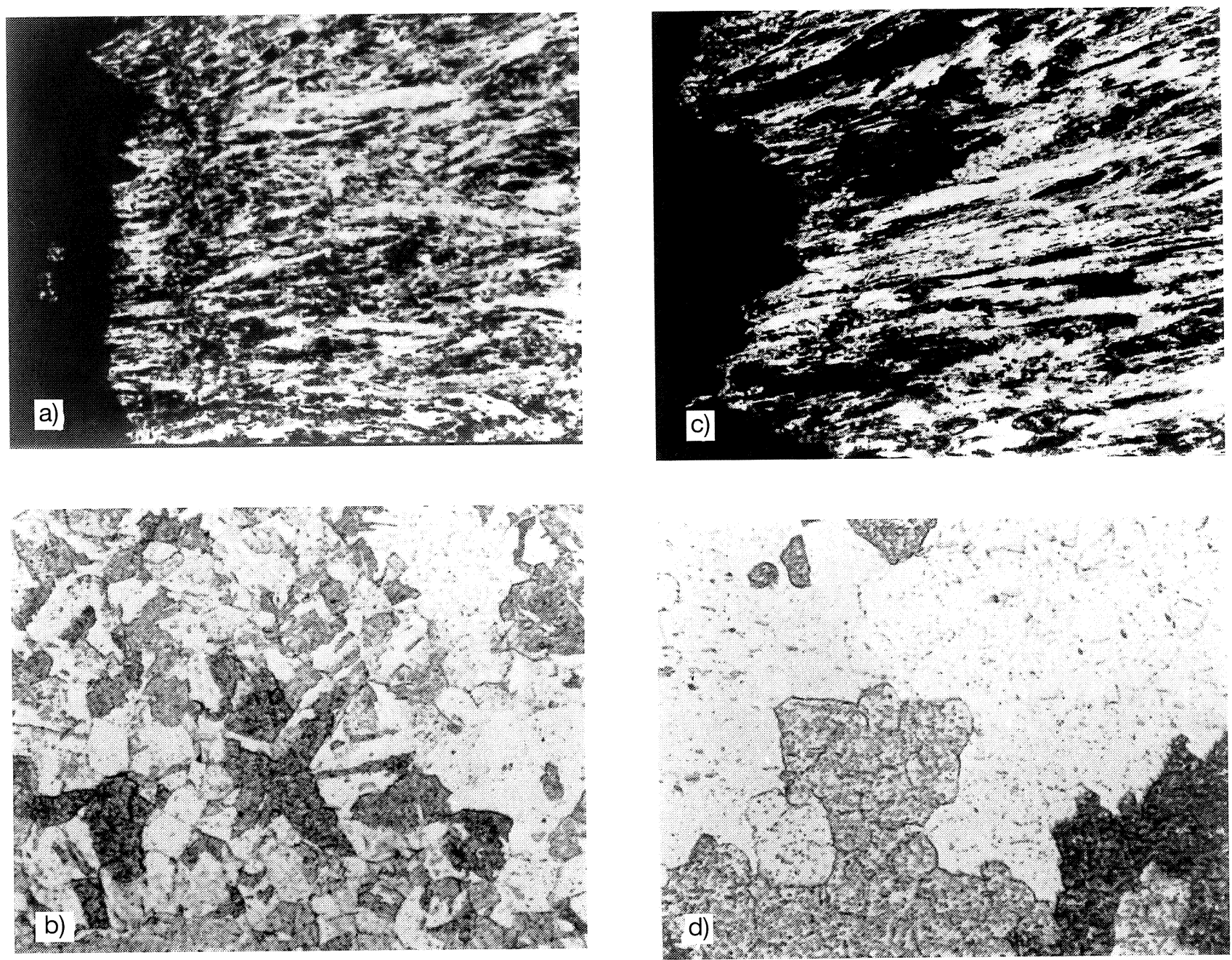

FIG. 17.- Microestructura del material 666 tras un ensayo de fluencia a $650{ }^{\circ} \mathrm{C}$. Nótese las diferencias en el modo de rotura.

a) y b) Duración del ensayo: $1.132 \mathrm{~h}$.

c) y d) Duración del ensayo: $6.314 \mathrm{~h}$.

FIG. 17. - Microstructure of the material 666 after a creep test at $650{ }^{\circ} \mathrm{C}$. Note the rupture mode difference.

a) and b) Test time: $1,132 \mathrm{~h}$.

b) and c) Test time: $6,314 \mathrm{~h}$.

activación, ya que se han conseguido aleaciones que pueden ser forjadas sin problemas, con valores mecánicos muy aceptables y muy poco activables. Aunque no llegan a alcanzar la resistencia a fluencia de las aleaciones MANET, se mantienen en el rango de las de baja activación (9).

- La adición de tantalio, además de reducir el tamaño de grano de la aleación 666, provoca una precipitación primaria de $\mathrm{TaC}_{3}$ dejando una matriz empobrecida de carbono. Como consecuencia, resulta un material inestable desde el punto de vista microestructural que sufre procesos de recristalización a temperatururas próximas a $600{ }^{\circ} \mathrm{C}$. Este hecho limitaría mucho su utilización como primera pared.
- La desoxidación con cerio ha resultado más eficaz que con itrio, por lo que hay un mayor exceso de nitrógeno en la aleación 664, y los valores de resistencia mecánica y resistencia a fluencia lenta son superiores que en la 667.

- Con el diseño propuesto para las aleaciones OPTIFER se ha conseguido la eliminación de ferrita $\delta$ de la microestructura.

\section{Agradecimiento}

El trabajo ha sido financiado por el Long-Term Fusion Technology Programme de las Comunidades Europeas. Los autores agradecen su colaboración a J. Serrano, L. Ramos, D. Plaza, V. Sanz, P. Graf y H. Zimmermann. 


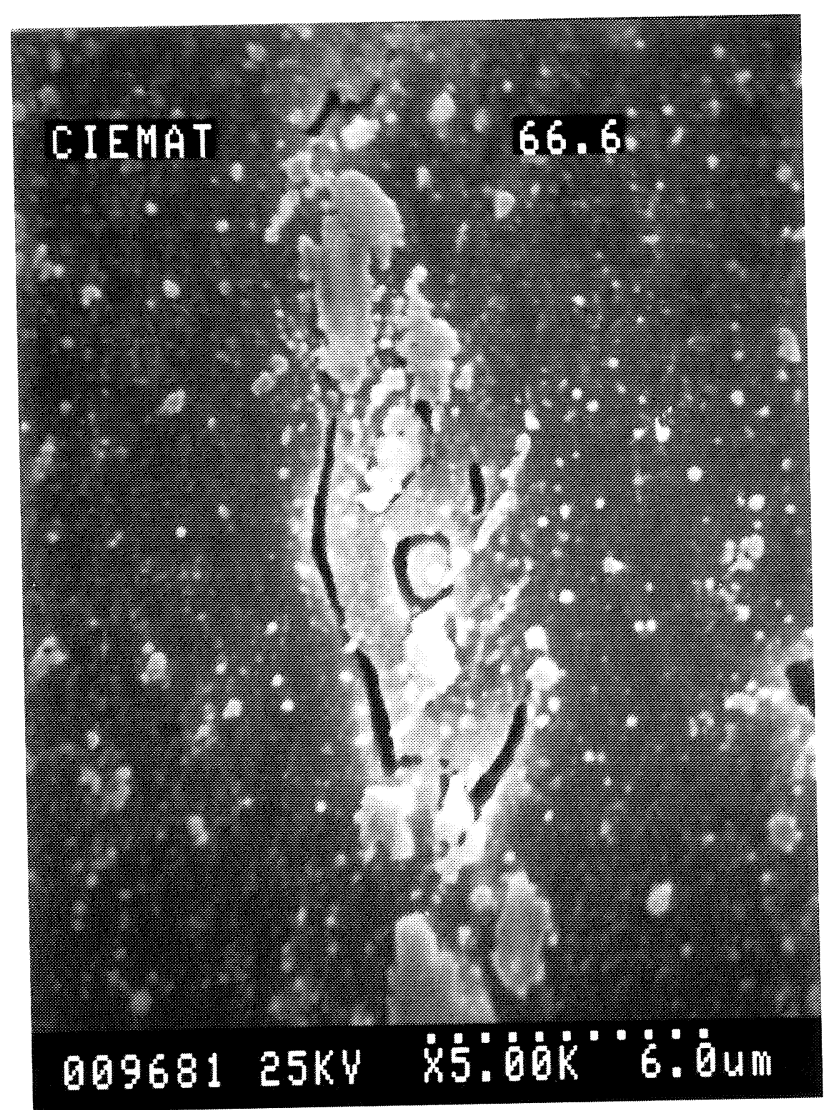

FIG. 18.- Microgrietas asociadas a los carburos de tantalio encontrados en el material 666. Zona próxima a la fractura por fluencia.

FIG. 18. - Microcracking associated with tantalum carbides precipitates found in the material 666. Nearest area to the creep fracture.

\section{REFERENCIAS}

(1) Weber, W.J., Mansur, L.K., Clinard, F. W. y Parkin, D.M. J. Nucl. Mater., 184, 1991: 1-21.

(2) Hishinuma, A. y Jitsukawa. S., J. Nucl. Mater., 169, 1989: 241-248.

(3) OKada. M., Noda. T. y AвE. F., J. Nucl. Mater., 169, 1989: 249-256.

(4) Lechtenberg, T., J. Nucl. Mater., 117, 1985: 133-134.

(5) LuCAS, G.E., J. Nucl. Mater., 216, 1994: 322-325.

(6) Kurishita, H., Kayano, H., Narui, M., Kimura, A., Hamilton, M.L. y Gelles, D.S. J. Nucl. Mater., 212-215, 1994: 730-735

(7) Schirra, M., Heger, S., Meinzer, H., Ritter, B. y SchWEIGER, W. Untersuchungen zum Vergütungsverhalten, Umwandlungsverhalten und der mechanischen Eigens-

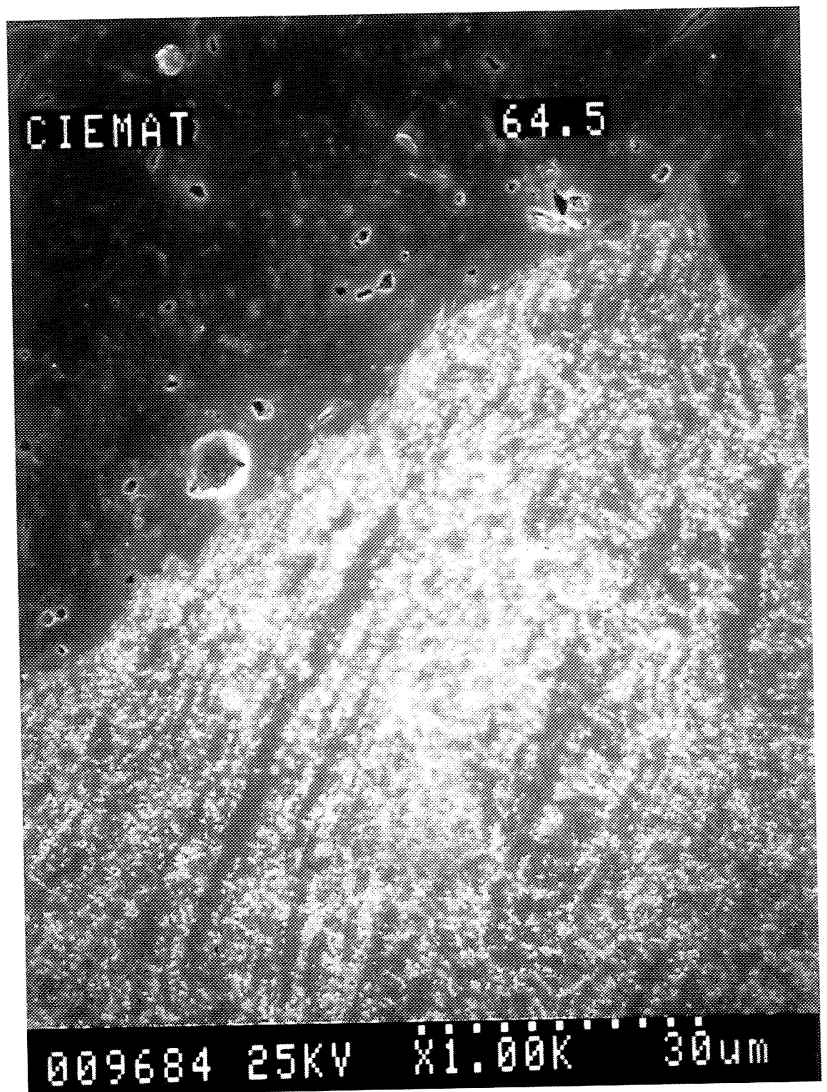

FIG. 19.- Material 664 tras un ensayo de fluencia. Nótese la ausencia de microgrietas en comparación con el material 666.

FIG. 19. - Material 664 after a crreep test. No microcracking can be found associated with the precipitates.

chaften am martensitischen Stahl 1.4914. (NET-Charge MANET-1). Kernforschungszentrum Karlsruhe. KfK 4561. Junio 1989.

(8) Schirra, M., Graf, P., Heger, S., Meimzer, H., SchweiGER, W. y ZIMMERMANN, H. MANET II: Untersuchungsergebnisse zum Umwandlungs- und Vergütungsverhalten und Prüfung Mechanischer Eigenschaften. Kernforschungszentrum Karlsruhe. KfK 5177. Mayo 1993.

(9) JARVIS, O.N. Low Activation Materials. Reuse and disposal, Harwell Report AERE-R 10860 (1983).

(10) Schirra, M., Schäfer, L., Heger, S., Meyer, W. y SchWEIGER, W. Untersuchungen an zwei Variaten neidrigaktivierender Martensitischer Stähle. 03/02/02P87A. Kernforschungszentrum Karlsruhe. Feb. 1990.

(11) Larson, F.R. y Miller. J. Trans, ASME, Jul. 1952: 765777. 